\title{
Structure and morphology studies of chromium film at elevated temperature in hypersonic environment
}

\author{
G M HEGDE*, V KULKARNI ${ }^{\ddagger}$, M NAGABOOPATHY ${ }^{\dagger}$ and K P J REDDY ${ }^{\dagger}$ \\ Centre for Nano Science and Engineering, ${ }^{\dagger}$ Department of Aerospace Engineering, Indian Institute of Science, \\ Bangalore 560 012, India \\ $\ddagger$ Department of Mechanical Engineering, Indian Institute of Technology, Guwahati, India
}

MS received 19 October 2009; revised 14 October 2011

\begin{abstract}
This paper presents the after shock heated structural and morphological studies of chromium film coated on hypersonic test model as a passive drag reduction element. The structural changes and the composition of phases of chromium due to shock heating $(2850 \mathrm{~K})$ are characterized using X-ray diffraction studies. Surface morphology changes of chromium coating have been studied using scanning electron microscopy (SEM) before and after shock heating. Significant amount of chromium ablation and sublimation from the model surface is noticed from SEM micrographs. Traces of randomly oriented chromium oxides formed along the coated surface confirm surface reaction of chromium with oxygen present behind the shock. Large traces of amorphous chromium oxide phases are also observed.
\end{abstract}

Keywords. Cr coating; Cr oxidation; drag reduction; surface morphology.

\section{Introduction}

Recent development in aerospace science has ushered in exciting era of hypersonic flights. Hypersonic flight involves extremely high velocities and the reduction of the wave drag encountered by such space vehicles is one of the major tasks in the hypersonic flight vehicle design. Various active techniques have been developed for the reduction of wave drag encountered by the space vehicle during its flight. Most of these techniques are centred on alteration of the flow field near the stagnation region. One such technique generally used for wave drag reduction is mounting of aerospike at the nose (Viren et al 2002) of the space plane to push the bow shock away from the surface of the body. Another technique based on similar concept is the injection of gas from the stagnation point (Venukumar et al 2006) to establish a fluid spike for drag reduction. Deposition of energy upstream of the stagnation point (Satheesh and Jagadeesh 2007) changes the flow encountered by the vehicle to reduce the drag. Marginal reduction in the drag force has also been observed by making the stepped afterbody (Viren et al 2005). Although these techniques have shown an appreciable reduction in the wave drag, their application to practical systems is inhibited due to their system complexity. In order to overcome this problem recently we have developed a passive drag reduction technique in which the drag reduction is achieved by adding heat into the shock layer in front of the blunt cone (Reding and Jecmen 1983; Srinivasan and Chamberlain 2004; Kulkarni et al 2008). Heat is added into the shock layer through the exothermic reaction of ablated atoms from the surface of the

\footnotetext{
*Author for correspondence (hegopal@aero.iisc.ernet.in)
}

model coated with about $10 \mu \mathrm{m}$ thick chromium. Another important aspect of future hypersonic systems is the need for high temperature materials for thermal protective systems (TPS). Chiang (1999) discussed in detail about $\mathrm{Cu}-\mathrm{Cr}$ based coating as protecting layers on aerodynamic model subjected to high enthalpy and high heat flux environments. Low oxidation rate materials (Opeka et al 2004) such as $\mathrm{SiO}_{2}, \mathrm{Al}_{2} \mathrm{O}_{3}$, $\mathrm{Cr}_{2} \mathrm{O}_{3}$ or $\mathrm{BeO}$ have been used in hypersonic TPS below $1800 \mathrm{~K}$ and $\mathrm{ZrB}_{2}$ and $\mathrm{HFB}_{2}$ were explored up to $2000 \mathrm{~K}$. Although these materials have been in use as TPS for decades for space vehicle models, the contribution of oxidation process of such coatings in wave drag reduction has not been studied. It is noted from the available literature that there is no experimental study of protective coatings simultaneously for the reduction of wave drag imposed on the vehicle by hypersonic flow.

The motivation for this investigation is to test the feasibility of chromium coating in wave drag reduction over a blunt body aerodynamic model (Kulkarni et al 2008) and hence provide insight into the microstructure, oxidation behaviour, and surface morphology relevant to chromium coatings in hypersonic environments. Previous studies have shown that oxidation behaviour of chromium is significantly different according to temperatures and the experimental parameters play an important role in chromium oxidation at high temperatures (Polman et al 1989; Buscail et al 2004). The temperature range explored in previous studies is up to $1800 \mathrm{~K}$. This paper reports on the structural and morphological studies of chromium coating as passive drag reduction element on a duralumin blunt cone model subjected to Mach 7 hypersonic flow conditions, attaining a freestream stagnation temperature of $2850 \mathrm{~K}$. 

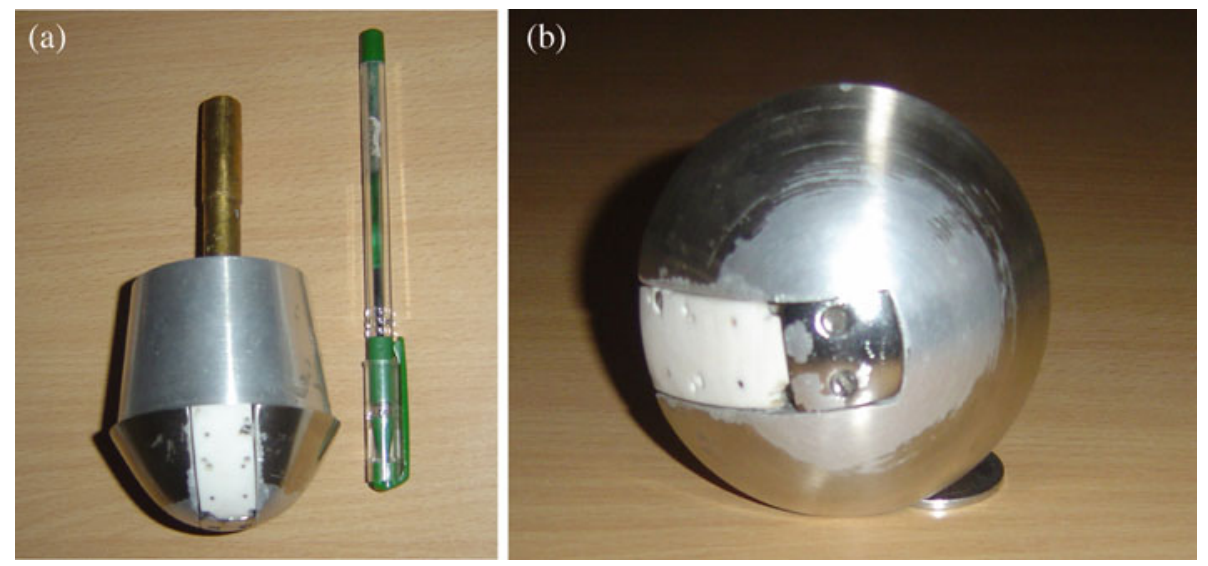

Figure 1. Chromium coated blunt cone model (a) top view and (b) side view.

\section{Experimental}

The experiment was conducted on a $60^{\circ}$ apex angle blunt cone model made of aluminium alloy, flying at hypersonic Mach number. The chromium coated model having $70 \mathrm{~mm}$ base diameter and bluntness ratio (defined by the ratio of nose diameter to base diameter) of 0.857 , is shown in figure 1. A sample piece $(1 \mathrm{~cm} \times 1 \mathrm{~cm} \times 5 \mathrm{~mm})$ of model material was flush mounted on the model, as shown in the figure, which was used for surface characterization of the model before and after shock exposure. Chromium is a hard metal with a high melting point of $2180 \mathrm{~K}$ and is an exothermic material having heat of vaporization, $339.5 \mathrm{~kJ} \cdot \mathrm{mol}^{-1}$. Chromium of about $95 \%$ purity was coated on the model surface using electrolytic deposition technique, to a thickness of typically $10 \mu \mathrm{m}$. Platinum thin film gauges coated on a $5 \mathrm{~mm}$ thick MACOR (thermally insulating material) were flush mounted on the model to measure heat flux on the wall of the model during hypersonic flow. The experiments on drag reduction study were carried out in the hypersonic shock tunnel (HST2), which consisted of a stainless steel shock tube of $50 \mathrm{~mm}$ internal diameter connected to a convergent divergent conical nozzle of $300 \mathrm{~mm}$ exit diameter. The driver and driven sections of the shock tube were separated by an aluminium diaphragm. Three pressure sensors were mounted along the length of the shock tube, on the driven section side, for the measurement of shock speed and the stagnation pressure at the entry to the nozzle. The Mach 7 hypersonic flow from the nozzle went through a $450 \mathrm{~mm}$ long test section of $300 \times 300 \mathrm{~mm}$ square cross-section. The specific freestream enthalpy of the flow in the shock tunnel was varied by choosing the metal diaphragm of appropriate thickness. The change in the drag coefficient was measured using an accelerometer based balance system and the heat addition due to the exothermic reactions was measured using surface mounted platinum thin film heat flux gauges and the corresponding displacement of the shock wave was measured using schlieren technique. Other details of the HST2 facility, experimental procedure and related calculations are reported in our earlier work (Viren et al 2002; Kulkarni et al 2008).

\section{Results and discussion}

The experiments were conducted at Mach 7 hypersonic flow of $3.4 \mathrm{MJ} / \mathrm{kg}$ enthalpy attaining a stagnation temperature of $2850 \mathrm{~K}$. Typical freestream conditions for the flow are given in table 1. In all our experiments the reaction time in the HST2 facility was typically $1 \mathrm{~ms}$, as estimated using the pitot pressure measurement. That means the surface gets heated to stagnation temperature for $1 \mathrm{~ms}$. The aerodynamics related results such as percentage drag reduction, drag coefficient, pressure and temperature measurements and flow field visualization around the model have already been published (Kulkarni et al 2008). The flush mounted test sample was used to characterize chromium coated model surface before and after shock heating. The structural and morphological changes of chromium surface subjected to hypersonic flow are studied using X-ray diffraction (XRD, Philips model-1070) and SEM (Cambridge Instruments Model-Stereo Scan360 and FEI, model-XC30Sirion). SEM images of the as-deposited chromium shown in figures 2(a) and (b) taken at $400 \times$ and $15000 \times$ orders of magnification, respectively reveal surface quality of the coating before shock exposure. It is clear from the figures that there was no uneven surface, not much variation in roughness and also no porosity as such. The spot like appearances seen in the $400 \times$ magnification are due to the image covering larger area including the bending region of the test specimen. X-ray diffraction pattern was taken using $\mathrm{CuK} \alpha$ irradiation to analyse the phases of as-deposited chromium on the

Table 1. Freestream conditions of current set of experiments in HST2 shock tunnel.

\section{$M_{\infty}$} 6.9

$P_{\infty}(\mathrm{kPa})$

0.654

$T_{\infty}(\mathrm{K})$

$321 \cdot 72$

Freestream stagnation pressure $(\mathrm{kPa})$ 4052

Freestream stagnation temperature $(\mathrm{K})$

Freestream stagnation enthalpy $(\mathrm{MJ} / \mathrm{kg}$ )
$2850 \cdot 28$

3.4 

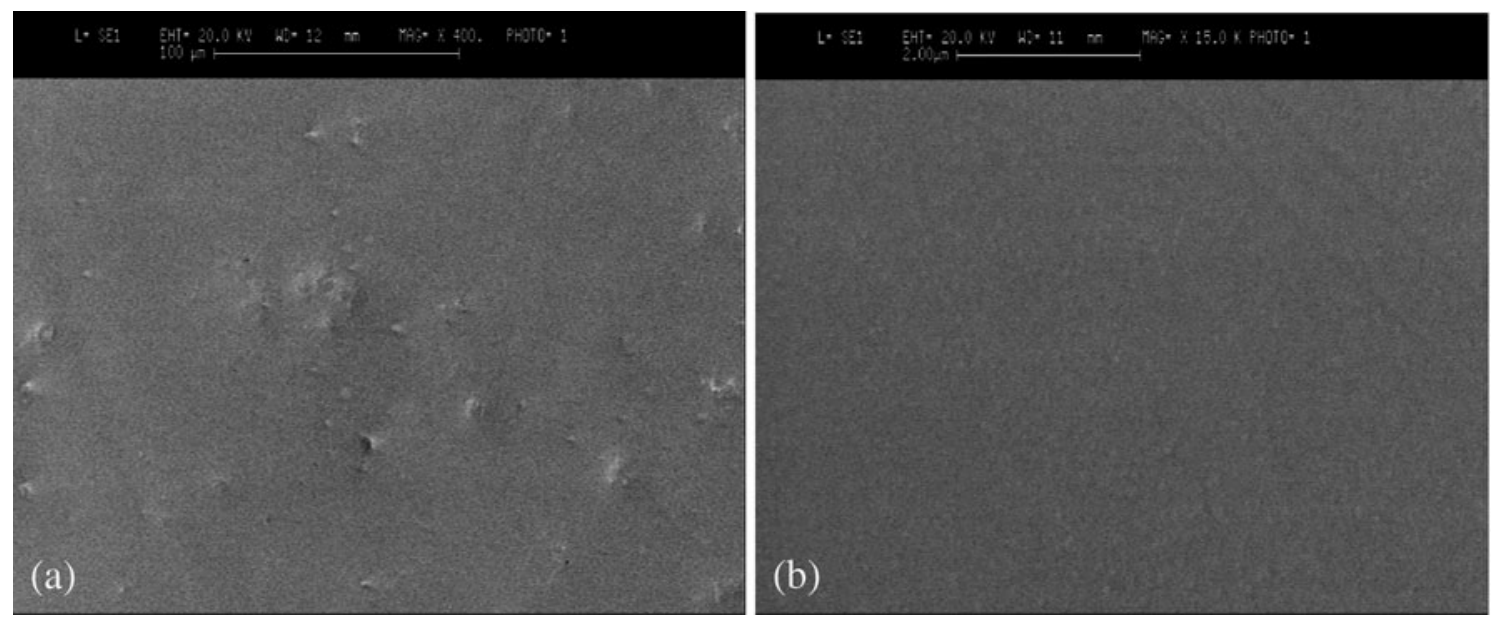

Figure 2. SEM micrographs of as-deposited chromium on model before shock exposure: (a) 400× magnification and (b) $15000 \times$ magnification.

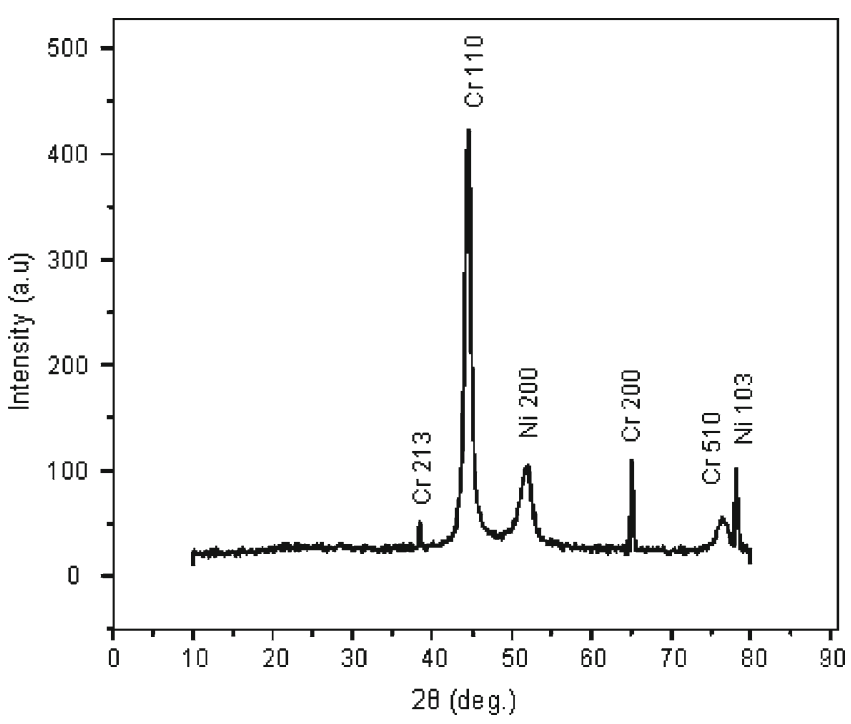

Figure 3. XRD pattern of chromium coating before shock exposure.

test piece and is shown in figure 3 . It shows a well defined peak at $2 \theta$ values of 44.5 degrees, followed by other peaks at 65.08 and 76.36 degrees. These are identified as diffraction from 110, 200 and 510 planes of body centred cubic (bcc) lattice. This pattern confirms that the chromium coating was most favourably $b c c$ structure prior to shock exposure. Angle chosen for this analysis is up to $90^{\circ}$ hence the higher order less intense peaks are not observed here. It is also observed that there are diffractions from the face centred cubic $(f c c)$ nickel planes like 200 and 103, which are present as impurities with chromium coating. As in the case of chromium, the higher angle less intense diffraction peaks are not observed for nickel as well. The $2 \theta$ range chosen in this analysis is to identify and confirm the high intense diffraction peaks of chromium and impurity nickel, which are the most favourable diffraction patterns widely reported for these materials.
Figures 4(a) and (b) show the post shock SEM images of surface morphology of the same regions of the sample taken before shock exposure. Figure 4(a) corresponds to the region of figure 2(a) but after shock heating, clearly showing that at certain regions chromium has been flushed out from the surface either due to sublimation or ablation, and roughness of the surface is increased abruptly. The kinetics of thermal sublimation of $\mathrm{Cr}$ at elevated temperature $(\sim 1100 \mathrm{~K})$ has been studied using low-energy electron microscopy (LEEM) (Tang et al 2006). They have reported that the surface of $\mathrm{Cr}$ begins to sublime at $\sim 1100 \mathrm{~K}$ at a slow but readily detectable rate. Three mechanisms of atomic layer removal from $\mathrm{Cr}$ surface have been discussed: (i) spontaneous nucleation and growth of two-dimensional vacancy islands, (ii) winding motions of single- and double-spiral steps that are pinned by bulk dislocations terminating at the surface and (iii) island (or mound) decay. These processes speed up as the temperature of the $\mathrm{Cr}$ crystal rises. Because of the step recession mechanism through which atomic layers are removed, the surface remains fairly smooth after many atomic layers are removed. All these mechanisms are observed in a slow heating process. However, in the present study, the heating process is very fast (few milliseconds) and duration of the steady state temperature $(2850.28 \mathrm{~K})$ is about $1.5 \mathrm{~ms}$ which is the test time of the hypersonic flow in our facility. This means, the material gets heated to this temperature for only about $1.5 \mathrm{~ms}$. Though the proposed mechanisms have certain role in $\mathrm{Cr}$ sublimation/ablation from the model surface, we could not observe these due to the limitations with our experimental conditions. Also, because of the high speed thermal process the surface will not remain smooth after the removal of few atomic layers which is clear from the SEM image (figure 4). Further, the ability of coating to withstand repetitive thermal shock without cracking is attributed to the close match of thermal expansion properties between $\mathrm{Al}$ alloy and $\mathrm{Cr}$.

Figure 4(b) corresponds to the region of figure 2(b) at $1600 \times$ magnification showing the flush out of chromium 

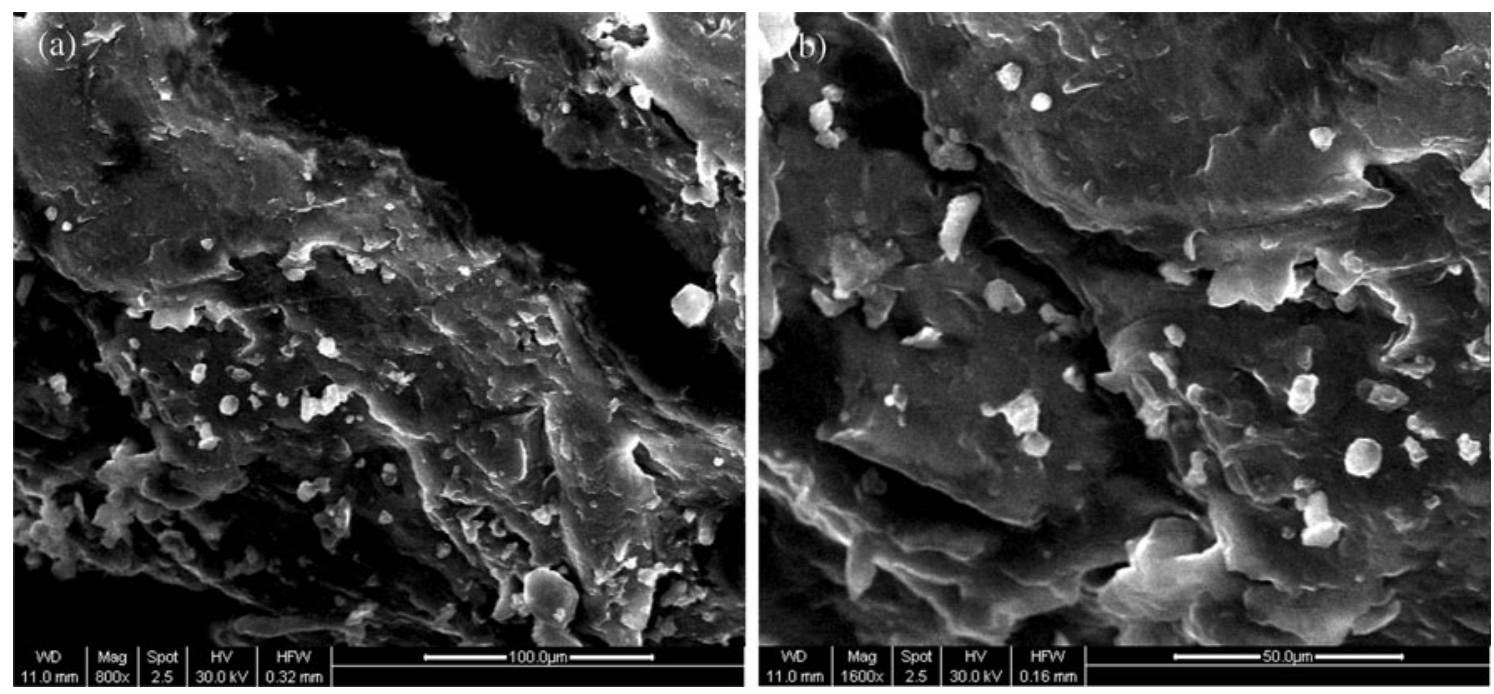

Figure 4. SEM images of chromium surface after high enthalpy shock exposure: (a) $800 \times$ magnification and (b) $1600 \times$ magnification.

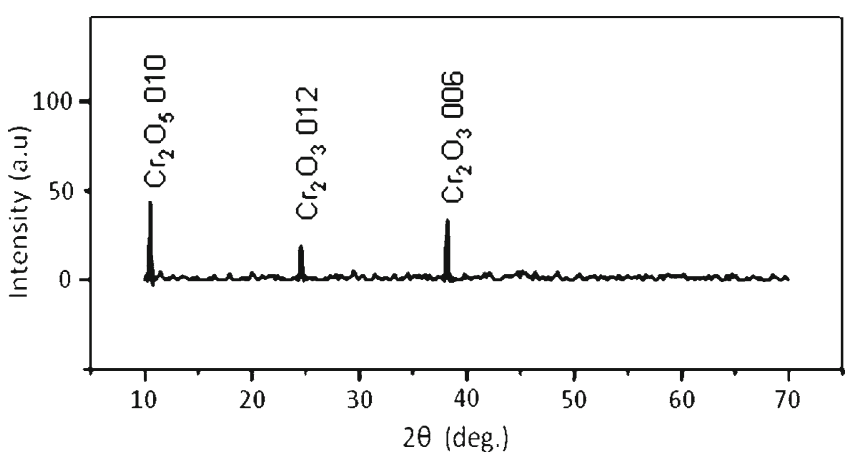

Figure 5. XRD pattern of chromium coating after high enthalpy shock exposure.

material on the surface at certain locations. After shock SEM images clearly show that top layers of $\mathrm{Cr}$ have been either sublimed or ablated and remaining part has reacted with the oxygen present in the hypersonic flow forming different $\mathrm{Cr}$ oxide phases. The white patches are the crystallized $\mathrm{Cr}$ oxide phases which are reflected in the XRD pattern (figure 5) whereas the remaining part is still an amorphous $\mathrm{Cr}$ oxide phase and are not seen in the after shock XRD. This amorphous structure is formed due to fast $(1.5 \mathrm{~ms})$ heating process which is the steady state high enthalpy flow condition of the facility. Because of the high thermal energy existing for a very short time, the entire coated surface exposed to the shock may not have sufficient thermal energy or time for complete crystallization. It is also reported that amorphousto-crystalline transformation of $\mathrm{Cr}$ oxide depends on the oxygen flux (Fu et al 2007) and in low oxygen flux $\mathrm{Cr}$ oxide phase is primarily amorphous (Xiaolu et al 2008). Distinguishable spherical bright aggregates over the surface are identified as oxides of chromium. X-ray analysis of the shock heated surface shown in figure 5 reveals that $b c c$ chromium reacted with the upcoming heat energy forming new high temperature reaction phases. Peaks observed at $2 \theta$ values, 24.58 and 38.9 degrees, correspond to the 012 and 006 crystal planes of rhombohedral crystal structure of $\mathrm{Cr}_{2} \mathrm{O}_{3}$ and peak at 10.56 corresponds to the diffraction of 010 monoclinic $\mathrm{Cr}_{2} \mathrm{O}_{5}$ phase. The results are in good agreement with previously reported data (Maslar et al 2001; Pang et al 2007, 2008). Impurity nickel peaks are not observed in the reacted phase as the material has ablated completely. Other oxide peaks are not seen in the diffraction pattern because of the randomness in oxide formation due to high speed, high enthalpy flow on conical surface of the model. Compared to the initial pre-shock peak intensity present in figure 3 , post shock intensities of the diffraction peaks appear to be weak. Such low S-to-N ratios of the diffraction patterns imply the existence of amorphous compounds. This is because at certain locations material was sublimed or ablated from the surface as observed from SEM micrographs and also oxidation has not taken place all over the surface but only at certain locations depending on the availability of the heat energy and oxygen atoms in the flow. The presence of substantial percentage of atomic oxygen ( 0.1536 mole fraction) in the shock layer is confirmed from the composition of the test gas used which is estimated using the computational code GASEQ for the tunnel operating conditions. The shock heated oxygen reacted with chromium forming the dispersed $\mathrm{Cr}_{2} \mathrm{O}_{3}$ and $\mathrm{Cr}_{2} \mathrm{O}_{5}$ which are the most favourable high temperature oxide layers to be formed for chromium (Polman et al 1989; Chiang 1999; Maslar et al 2001; Buscail et al 2004; Opeka et al 2004; Pang et al 2007, 2008). Thus the measured reduction of drag force is due to the release of heat energy into the shock layer by the exothermic reaction of chromium atoms on the model surface with the atomic oxygen present in the shock layer of the high enthalpy flow. Use of chromium coating as TPS due to its low oxidation rate at high temperatures has already been demonstrated (Chiang 1999; Opeka et al 2004). 
In summary, the $\mathrm{Cr}$ coated test model exposed to high enthalpy shock wave generated by hypersonic flow will undergo high shearing action and local heating over the surface leading to material sublimation and ablation. Part of the energy is utilized in chromium oxide formation both in amorphous and crystalline phases, reacting with the oxygen atoms present in the high enthalpy flow. This process helps stop the propagation of energy into inner layer of the coating and hence protecting the model surface from any thermal damage. Thus chromium coating protects the model from direct thermal damage by utilizing the upcoming heat energy in changing its crystal phase, partial oxidation with oxygen present in the free stream and ablation of material from the surface.

\section{Conclusions}

The oxidation and morphological studies of chromium coating on aluminium alloy hypersonic test model subjected to Mach 7 hypersonic flow conditions attaining a temperature of $2850 \mathrm{~K}$ are presented. After shock SEM analysis of the coating shows that there is considerable amount of chromium sublimation/ablation at certain regions of the surface due to shock heating. XRD analysis reveals that at certain regions of the surface, the pre-shock bcc chromium phases are transformed into dispersed rhombohedral structures of chromium oxide phase of $\mathrm{Cr}_{2} \mathrm{O}_{3}$ and traces of monoclinic $\mathrm{Cr}_{2} \mathrm{O}_{5}$ phase. Other regions with amorphous $\mathrm{Cr}$ oxide phases having rough surface are also seen. The observed drag reduction is attributed to the surface reaction of chromium film with high energy flow adding heat into the shock layer. Hence chromium coating on hypersonic models can serve the dual purpose of wave drag reduction as well as thermal protection layer.

\section{Acknowledgements}

The authors would like to thank DRDO for funding. We also thank all members of Laboratory for Hypersonics and Shock Waves Research for their help and support.

\section{References}

Buscail H, Jacob Y P, Stroosnijder M F, Caudron E, Cueff R, Rabaste F and Perrier S 2004 Mater. Sci. Forum 461-464 93

Chiang K T 1999 Surf. Coat. Technol. 1141

Fu C M, Lai C J, Hsu H S, Chao Y C, Huang J C A, Wu C -C and Shyu S -G 2007 J. Mater. Res. 223531

Kulkarni V, Hegde G M, Jagadeesh G, Arunan E and Reddy K P J 2008 Physics of Fluids 20081703

Maslar J E, Hurst W S, Vanderah T A and Levin I 2001 J. Raman Spectrosc. 32201

Opeka M M, Talmy I G and Zaykoski J A 2004 J. Mater. Sci. 39 5887

Pang X, Gao K and Volinsky A 2007 J. Mater. Res. 223531

Pang X, Gao K, Luo F, Yang H, Qiao L, Wang Y, Alex A and Volinsky A 2008 Thin Solid Films 5164685

Polman E A, Fransen T and Gellings P J 1989 Oxidation of Metals 32433

Reding J P and Jecmen D M 1983 J. Spacecraft and Rockets 20452

Satheesh K and Jagadeesh G 2007 Physics of Fluids 19031701

Srinivasan G R and Chamberlain R R 2004 AIAA 47141

Tang S -J, Kodambaka S, Swiech W, Petrov I, Flynn C P and Chiang T -C 2006 Phys. Rev. Lett. 96126106

Venukumar B, Jagadeesh G and Reddy K P J 2006 Physics of Fluids 18118104

Viren M, Saravanan S and Reddy K P J 2002 Shock Waves 12197

Viren M, Kumar S, Maruta T, Reddy K P J and Takayama K 2005 Shock Waves 14421

Xiaolu P, Kewei G, Fei L, Huisheng Y, Lijie Q, Yanbin W, Alex A and Volinsky A 2008 Thin Solid Films 5164685 